\title{
Simulation of the dynamics of an intense proton beam in a collider NICA
}

\author{
Margarita Korobitsina ${ }^{1 *}$, Alexander Kovalenko ${ }^{1}$, and Andrey Kolomiets ${ }^{1,2}$ \\ ${ }^{1}$ Join Institute of Nuclear Research, 141980 Dubna, Russia \\ ${ }^{2}$ Institute for Theoretical and Experimental Physics of National Research Centre "Kurchatov \\ Institute", 117218 Moscow, Russia
}

\begin{abstract}
In the physics of accelerators and colliders there is one important parameter as "impedance". Impedance determines the level of the influence of the field induced by the beam in the longitudinal and transverse directions on the beam motion. Knowledge of the impedances is necessary for estimating the conditions for the stability of the beam motion in the designed accelerator. Thus, the main objective at the moment is the calculation of the impedance of each device in the collider for further calculation of their contribution to the general impedance. In the report the physics of the beam motion in an accelerator chamber is considered, the method of calculating the impedance - the method of electro-magnetic simulation through the CST Studio Suit software package is described, an example of the calculation of the impedance for the part of vacuum chamber of the quadrupole magnet, which is a part of the arch of the collider NICA is shown.
\end{abstract}

\section{Introduction}

An intense beam of charged particles, which moves in the vacuum chamber of the accelerator, induces electromagnetic fields (wake-fields) in the walls of the structure elements. The appearance of resonance conditions between the frequency of the bunch revolution and the frequency of the wake field, leads to an increase in the amplitude of the oscillations (instability of the longitudinal and transverse motion). The study of this phenomenon led to the introduction of the concept of impedance, which determines the level of field influence on the beam in the accelerator in the longitudinal and transverse direction.

\section{Physics of the beam motion}

Let us consider a beam circulating inside an accelerator device as shown in Figure 1.

\footnotetext{
*Corresponding author: telegina_m95@mail.ru
} 


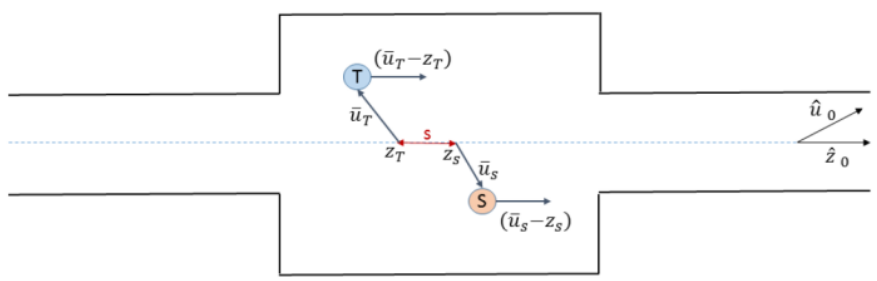

Fig. 1: Scheme of the source (S) and test (T) particle for impedance calculation [1].

Figure 1 shows a source particle (S) and a test particle (T) that are travelling along the axis of a beam chamber. The source (S) particle excites an electromagnetic field that acts back on the test (T) particle [1].

In order to describe the problem, it is necessary to adopt a cylindrical coordinate system, where $\hat{u}=u \hat{u}_{0}$ - is the transverse coordinate, $\hat{z}=z \hat{z}_{0}$ - is the longitudinal coordinate. The test particle is positioned at $\left(\hat{u}_{T}, z_{T}\right)$, and $s$ - is the distance to the source particle position $\left(\hat{u}_{S}, z_{S}=\beta c t\right)[1]$.

To obtain information about the scattered field impact on longitudinal and transverse dynamics, the change in transverse and longitudinal momentum of the test particle can be calculated, if the velocity of the source and the test particle is constant along the device.

The change of momentum is given by the equation [1]:

$$
\Delta \bar{p}\left(\bar{u}_{S}, \bar{u}_{T}, s\right)=\int_{-\infty}^{+\infty} \bar{F}\left(\bar{u}_{S}, \bar{u}_{T}, z_{T}=z_{S}-s, z_{S}\right) d t
$$

where $\bar{F}=q\left(\bar{E}+\beta \mu_{0} c \hat{z}_{0} * \bar{H}\right)-$ is the general force, $\mu_{0}$ - is the vacuum permeability, $q$ - is the particle charge. The projection components of the momentum variation in the longitudinal and transverse plane are given by the equations [1]:

$$
\begin{gathered}
\Delta p_{l}\left(\bar{u}_{S}, \bar{u}_{T}, s\right)=\int_{-\infty}^{+\infty} E_{l}\left(\bar{u}_{S}, \bar{u}_{T}, z_{T}\right) d t \\
\Delta p_{t}\left(\bar{u}_{S}, \bar{u}_{T}, s\right)=q \int_{-\infty}^{+\infty} \hat{u}_{0} * E_{l}\left(\bar{u}_{S}, \bar{u}_{T}, z_{T}, z_{S}\right)+\beta \mu_{0} c \hat{z}_{0} * H_{t}\left(\bar{u}_{S}, \bar{u}_{T}, z_{T}, z_{S}\right) d t
\end{gathered}
$$

where $z_{S}=\beta c t, z_{T}=z_{S}-s$.

Finally, the longitudinal and transverse wake functions can be defined as [1]:

$$
\begin{aligned}
& W_{l}\left(\bar{u}_{S}, \bar{u}_{T}, s\right)=-\frac{\beta c}{q Q} \Delta p_{l}\left(\bar{u}_{S}, \bar{u}_{T}, s\right) \\
& W_{t}\left(\bar{u}_{S}, \bar{u}_{T}, s\right)=-\frac{\beta c}{q Q} \Delta p_{t}\left(\bar{u}_{S}, \bar{u}_{T}, s\right),
\end{aligned}
$$

where $q$ - is the charge of the test particle, $Q$ - is the charge of the source particle.

The beam coupling longitudinal and transverse impedance is defined as the Fourier transform of the longitudinal and transverse wake-field, respectively [1]:

$$
\begin{gathered}
Z_{l}\left(\bar{u}_{S}, \bar{u}_{T}, \omega\right)=\int_{-\infty}^{+\infty} W_{l}\left(\bar{u}_{S}, \bar{u}_{T}, s\right) e^{\frac{j w s}{\beta c} \frac{d s}{\beta c}} \\
Z_{t}\left(\bar{u}_{S}, \bar{u}_{T}, \omega\right)=-j \int_{-\infty}^{+\infty} W_{t}\left(\bar{u}_{S}, \bar{u}_{T}, s\right) e^{\frac{j w s}{\beta c}} \frac{d s}{\beta c}
\end{gathered}
$$




\section{Electromagnetic simulations}

Calculation of impedances at the design stage of the structure nodes of the accelerator complex allows estimating the quality of design solutions and contributes to the achievement of optimal design results. As the simulation results accumulate, it becomes possible to determine the complete model of the coupling impedance of the collider structure.

One of the most suitable for this task is the CST Studio Suit software package. Section CST PARTICLE STUDIO (CST PS) is designed for full sequential modeling of free moving charged particles. The program was widely used to calculate the impedance at CERN accelerators. The results of these calculations were experimentally confirmed.

The standard output of the program is the wake potential: $W_{\|}-$longitudinal, $W_{\perp}$ transverse. The computation of the longitudinal component even simplifies to an integration of $E_{z}$ since the magnetic fields do not contribute to this component [2]:

$$
W_{\|}\left(r_{1}, s\right)=\frac{1}{q_{1}} \int_{-\infty}^{\infty} d z\left[\mathrm{E}_{z}\left(r_{1}, z_{s}, t\right)\right]_{t=(s+z) / c}
$$

If the tangential components of the electric field vanish on the integration boundaries, the so called Panofsky - Wenzel theorem can be used to compute the transverse wake components directly from the longitudinal one [2]:

$$
W_{\perp}\left(r_{1}, s\right)=-\nabla \int_{-\infty}^{s} d s^{\prime} W_{\|}\left(\left(r_{1}, s\right)\right.
$$

The longitudinal wake impedance is computed by the Fourier-transformation of the longitudinal component of the wake potential, divided by the Fourier-transformed charge distribution function $\lambda(\mathrm{s})[2]$ :

$$
Z_{||}(\omega)=\frac{\int_{-\infty}^{\infty} W_{\|}(s) e^{-i \omega s} d s}{\int_{-\infty}^{\infty} \lambda(s) e^{-i \omega s} d s}
$$

The transversal wake impedance is given by [2]:

$$
Z_{\perp}(\omega)=i \frac{\int_{-\infty}^{\infty} W_{\perp}(s) e^{-i \omega s} d s}{\int_{-\infty}^{\infty} \lambda(s) e^{-i \omega s} d s}
$$

In this way it is possible to determine the dipole and quadrupole components only for an axially symmetric element.

For elements of a structure that do not have symmetry, the components of the transverse impedance can be determined by applying the method outlined in Ref. [3] with the use of post processing macros CST Studio.

\section{Calculating of the impedance}

Let us consider the results of calculating of the impedances using the example of a vacuum chamber of a quadrupole magnet, which will be used in a collider NICA.

A 3D model of the vacuum chamber of a quadrupole magnet is shown in Figure 2. 


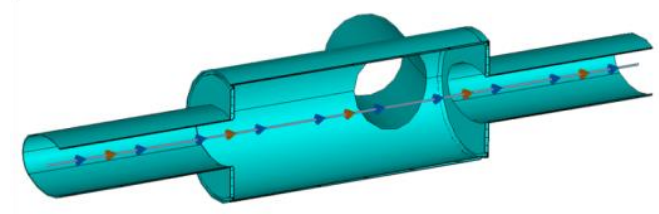

Fig. 2: 3D model of the quadrupole magnet camera.

The chamber is a connection of a segment of an elliptical chamber (semi-major axis $r_{x}=$ $61 \mathrm{~mm}$, semi-minor axis $r_{y}=36 \mathrm{~mm}$ ) similar to a dipole magnet chamber, with a cylindrical part (radius $R=80 \mathrm{~mm}$ ) intended for housing a branch pipe (radius $r=54 \mathrm{~mm}$ ) that connects the vacuum chamber to the vacuum pump. The structural heterogeneities associated with transitions from elliptical geometry to cylindrical and backward are the sources of the coupling impedances of a beam in a given element of the collider structure.

The result of modeling of the longitudinal impedance of the quadrupole magnet chamber is shown in Figure 3.

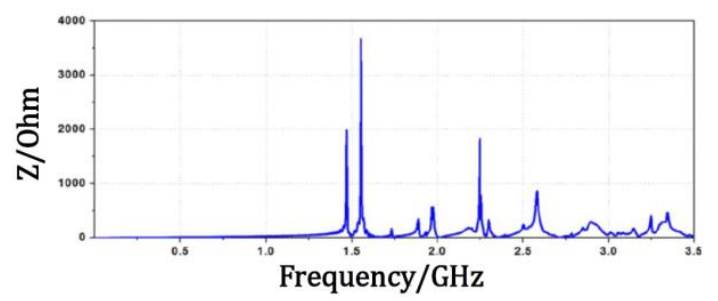

Fig. 3: Longitudinal impedance (absolute value) of the quadrupole magnet chamber.

Impedance is characterized by the presence of a number of high-frequency resonances, whose parameters are determined by the geometry of the chamber. The maximum value of the longitudinal impedance does not exceed $4 \mathrm{k} \Omega$. In the frequency range from 0 to $1,2 \mathrm{GHz}$, the value of the longitudinal impedance varies monotonically from 0 to $50 \mathrm{Ohm}$.

The distributions of the electric field in the chamber for several frequencies are shown in Figure 4. The figure shows that at all modes the electric field induced by the beam is concentrated in the cylindrical part of the chamber. This phenomenon can easily be eliminated by using a screen with a high vacuum conductivity to shield the cylindrical part of the chamber.

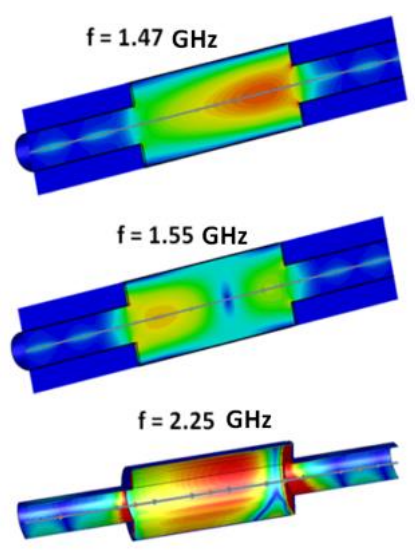

Fig. 4: Distributions of the electric field in the chamber of a quadrupole magnet at different frequencies. 
The transverse impedances of the quadrupole magnet chamber are shown in Figure 5.
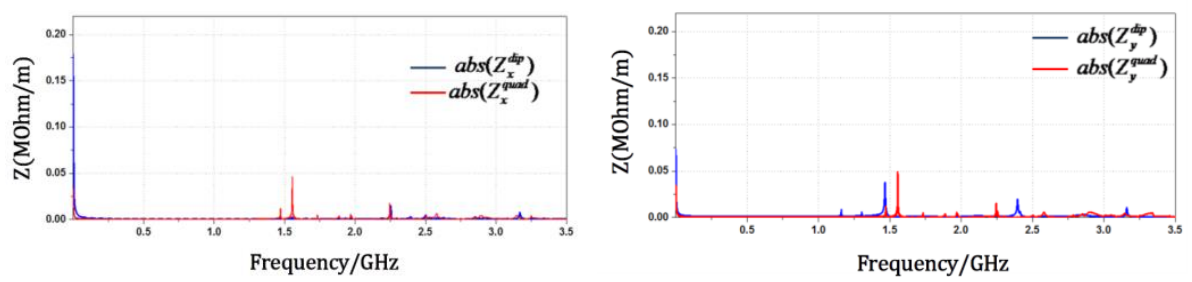

Fig. 5: Transverse impedance of the quadrupole magnet chamber. The left figure shows the XOZ plane: the blue curve represents the absolute value of the impedance $Z_{x}^{\text {dip }}$, red $-Z_{x}^{\text {quad }}$. The right figure shows the YOZ plane: the blue curve represents the absolute value of the impedance $Z_{y}^{d i p}$, red - $Z_{y}^{\text {quad }}$.

Simulation of longitudinal and transverse impedances was carried out with Wakefield Solver parameters given in Table 1.

Table 1. Wakefield Solver parameters used in modeling

\begin{tabular}{|c|c|}
\hline Bunch length, rms & 30 \\
\hline Integration distance, $\mathrm{m}$ & 10 \\
\hline Frequency max, GHz & 3,5 \\
\hline Bunch charge, $\mathrm{K}$ & $1 \cdot 10^{-9}$ \\
\hline
\end{tabular}

\section{Conclusions}

The resonance peaks obtained in the frequency spectrum of the longitudinal and transverse components of the wake potential make it possible to analyze both the cause of their appearance and the potential danger from the point of view of the effect of these fields on the perturbation of particle motion in the collider.

The results are considered as a first stage for future simulation of the dynamics of a beam. The actual task for today is to analyze all the elements that make up the NICA accelerator complex which directly contribute to the impedance. Thus, having knowledge about the dynamics and the instability of the beam in the accelerator/collider, it will be possible to set tasks for optimization of design solutions in order to minimize impedances and obtain the largest luminosity of the collider.

\section{References}

1. S. Persichelli, "The Beam Coupling Impedance Model of the CERN Proton Synchrotron”, PhD thesis, (University of Rome La Sapienza, 2015), p. 10-12

2. CST STUDIO SUITE http://www.cst.com

3. C. Zannini, K.Li, G. Rumolo, Effects of an asymmetric chamber on the beam coupling impedance, (Proceedings of IPAC2012, New Orleans, Louisiana, USA, 2012), p. 3099 